\section{Introducing a new alcohol-free hand disinfectant foam}

Dentisan are delighted to introduce a new non-alcohol post wash skin disinfectant foam to their product portfolio. New DentiSure is an important addition to Dentisan's already comprehensive range of hand care products and dispensing solutions, providing a non-alcohol alternative for those who prefer this option.

Hand hygiene is one of the most important measures in reducing the risk of infection transmission and all dental practices have a duty of care to ensure they are managed in accordance with current regulations and guidelines regarding hand hygiene. DentiSure makes an important contribution to this work, being fully compliant with HTM 01-05 and having been microbiologically tested to British Standard BS EN 1500.

DentiSure is supplied in a 1 litre ready-to-use format and should

be applied to the hands after washing and drying, and rubbed in until fully dispersed. It can also be used on clean skin between washes to minimise pathogen transmission. This complete coverage of the exposed skin provides additional protection from pathogens for those working within dental practices.

DentiSure is available in a ready to use 1 litre foam pump dispenser, and can be ordered exclusively from Henry Schein Dental on 08000232558 and Kent Express on 01634 878787. www.dentisan.co.uk

\title{
Get support with your stock investments
}

Are you looking to invest into the stock market? Then there are a few factors that you need to take into consideration.

Firstly, what kind of stock are you looking to purchase? As well as the two main classes - common and preferred - companies sometimes offer customised shares, which are often broken down into groups.

Each type is different, so if you're not entirely sure what kind of stock you're looking for that will help achieve your goals, it is best to seek guidance from an Independent Financial Adviser.

Other areas to think about include how many shares you intend to buy (or sell), the amount you're willing to pay and how you're going to deal.

There are a number of different ways you can go about it, but for minimum hassle and to achieve maximum return with minimal losses, it is always best to use the services of an expert.

At money4dentists, the highly experienced team of Independent Financial Advisers offer an advisory service to assist you in the buying of your shares.

If you would like to find out more, contact money4dentists today on 0845345 5060, or email info@money4dentists.com.

\section{Arm your implant patients}

More and more patients are choosing implants. They fit and function like natural teeth and lack the stigma of dentures.

Caring for implants is important and your patients must arm themselves with the best tools. Tandex has produced a high-quality range of brushes and adjunctive products, including some that can be used by implant patients on a daily basis.

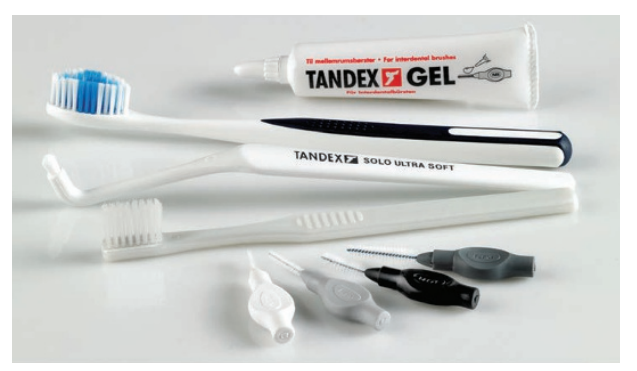

The SOLO interspace brush has been specially designed for critical points, such as deep periodontal pockets, post-surgery and around implants. It will remove debris gently, yet effectively helping the implant to stay healthy and stable for longer.

It is just one product in the Tandex range of brushes that are suitable for all age groups and dental needs. Ergonomically designed, they provide professional-level cleaning every day.

The SOLO interspace is complemented by the FLEXI and the PROXI interdental brushes for cleaning in all of the hard-to-reach places.

Implants are only the 'miracle' solution if the treatment is supported by comprehensive aftercare. Talk to your implant patients about Tandex and they can enjoy stable implants for longer.

For more information on Tandex's range of products visit www.tandex.dk.

\section{Ensure your patients are comfortable and reassured}

The team at EndoCare is absolutely committed to providing all of its patients with stunning care - which is why they are excited to announce their latest investment: the FollowApp.

This intuitive service will allow us to closely monitor our patients during their home recovery, ensuring that they are always comfortable and reassured. Patients are sent an SMS message containing a simple, one-minute survey -the responses to which will keep us informed of their recovery. This way, we can offer them advice and reassurance or, if necessary, call them back in for follow up treatment.

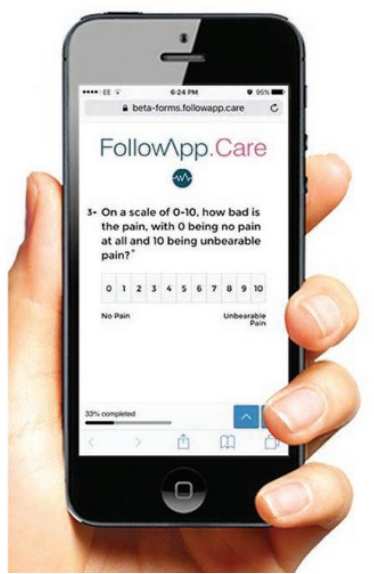

The app is a university-tested system and is showing great promise. EndoCare feels this is a modern way of letting patients know that we are fully supporting them from the beginning of their treatment to the very end.

EndoCare is the first endodontic practice in the UK to be using this system, and believe it will be of great benefit to patients and referring colleagues.

To find out more about referring your endodontic cases to one of the UK's most trusted referral centres, contact the team today.

For further information call EndoCare on 02072240999 or visit www.endocare.co.uk. 